\title{
Emulsion detectors for the antihydrogen detection in AEgIS
}

\author{
C. Pistillo ${ }^{1}$-S. Aghion ${ }^{2,3}$. C. Amsler ${ }^{1}$ - A. Ariga ${ }^{1}$. T. Ariga ${ }^{1}$ - A. Belov ${ }^{4}$. \\ G. Bonomi ${ }^{5,6}$ • P. Bräunig ${ }^{7}$. J. Bremer ${ }^{8}$. R. S. Brusa9, ${ }^{9} 10$ - L. Cabaret ${ }^{11}$.
}

M. Caccia ${ }^{3,12}$ - R. Caravita ${ }^{13,14}$ - F. Castelli ${ }^{3,15}$ - G. Cerchiari ${ }^{16}$ - K. Chlouba ${ }^{17}$.

S. Cialdi ${ }^{3,15}$. D. Comparat ${ }^{11}$ - G. Consolati ${ }^{2,3}$ - A. Demetrio ${ }^{7}$ - H. Derking ${ }^{8}$.

L. Di Noto ${ }^{13,14} \cdot$ M. Doser ${ }^{8}$ - A. Dudarev ${ }^{8}$. A. Ereditato ${ }^{1}$ - R. Ferragut ${ }^{2,3}$.

A. Fontana ${ }^{6} \cdot$ S. Gerber ${ }^{8}$. M. Giammarchi ${ }^{3}$ - A. Gligorova ${ }^{18}$. S. Gninenko ${ }^{4}$.

S. Haider ${ }^{8}$. S. Hogan ${ }^{19}$ • H. Holmestad ${ }^{20}$ T. Huse ${ }^{20}$ - E. J. Jordan ${ }^{16}$.

J. Kawada $^{1}$ - A. Kellerbauer ${ }^{16}$ • M. Kimura ${ }^{1}$ D. Krasnický ${ }^{13,14}$.

V. Lagomarsino ${ }^{13,14} \cdot$ S. Lehner ${ }^{21}$. C. Malbrunot ${ }^{8,21} \cdot$ S. Mariazzi ${ }^{21}$.

V. Matveev ${ }^{4}$ Z Z. Mazzotta ${ }^{3,15} \cdot$ G. Nebbia ${ }^{22}$ - P. Nédélec ${ }^{23} \cdot$ M. Oberthaler ${ }^{7}$.

N. Pacifico ${ }^{18} \cdot$ L. Penasa ${ }^{9,10} \cdot$ V. Petráček ${ }^{17} \cdot$ F. Prelz ${ }^{3} \cdot$ M. Prevedelli ${ }^{24}$.

L. Ravelli, ${ }^{910}$. C. Riccardi ${ }^{6,25}$. O. Røhne ${ }^{20}$. S. Rosenberger ${ }^{8}$ - A. Rotondi ${ }^{6,25}$.

H. Sandaker ${ }^{18}$. R. Santoro ${ }^{3,12}$. P. Scampoli ${ }^{1,26} \cdot$ M. Simon ${ }^{21} \cdot$ M. Śpaček ${ }^{17}$.

J. Storey ${ }^{1}$ I. M. Strojek ${ }^{17}$ - M. Subieta ${ }^{5,6}$. G. Testera ${ }^{14}$. E. Widmann ${ }^{21}$.

P. Yzombard ${ }^{11} \cdot$ S. Zavatarelli ${ }^{14} \cdot$ J. Zmeskal ${ }^{21}$

Published online: 16 April 2015

(C) Springer International Publishing Switzerland 2015

Abstract The AEgIS experiment at CERN aims to perform the first direct measurement of gravitational interaction between matter and antimatter by measuring the deviation of a cold antihydrogen beam in the Earth gravitational field. The design of the experiment has been recently updated to include emulsion films as position sensitive detector. The submicrometric position accuracy of emulsions leads indeed to a significant improvement of the experimental sensitivity. We present results of preliminary tests and discuss perspectives for the final measurement.

Proceedings of the International Conference on Exotic Atoms and Related Topics (EXA 2014), Vienna, Austria, 15-19 September 2014

C. Pistillo

ciro.pistillo@cern.ch

1 Albert Einstein Center for Fundamental Physics, Laboratory for High Energy Physics,

University of Bern, 3012 Bern, Switzerland

2 Politecnico of Milano, Piazza Leonardo da Vinci 32, 20133 Milano, Italy

3 INFN Milano, via Celoria 16, 20133 Milano, Italy

4 Institute for Nuclear Research of the Russian Academy of Science, Moscow 117312, Russia 
Keywords Emulsions · Antimatter A Antihydrogen

\section{Introduction}

Emulsion-based detectors always contributed to the history of nuclear and particle physics with fundamental discoveries and measurements that profited from their unsurpassed spatial and angular resolution in the measurement of charged particle tracks. Despite the

5 Department of Mechanical and Industrial Engineering, University of Brescia, via Branze 38, 25123 Brescia, Italy

6 INFN Pavia, via Bassi 6, 27100 Pavia, Italy

7 Kirchhoff-Institute for Physics, Heidelberg University, Im Neuenheimer Feld 227, 69120 Heidelberg, Germany

8 Physics Department, CERN, 1211 Geneva 23, Meyrin, Switzerland

9 Department of Physics, University of Trento, via Sommarive 14, 38123 Trento, Italy

10 TIFPA/INFN Trento, via Sommarive 14, 38123 Trento, Italy

11 Laboratory Aim Cotton, CNRS, University of Paris-Sud, ENS Cachan, Bt. 505, 91405 Orsay, France

12 Department of Science, University of Insubria, Via Valleggio 11, 22100 Como, Italy

13 Department of Physics, University of Genova, via Dodecaneso 33, 16146 Genova, Italy Complesso Universitario di Monte S.Angelo, 80126 Napoli, Italy 
decline of the technique around 1960-70 due to the development and use of the modern electronic particle detectors, emulsions are still used today, thanks to the vigorous rebirth of the technique that took place around the beginning of the 1990s, driven by the needs of neutrino experiments. The realization of industrial production chains for large scale emulsion detector manufacturing and the development of fully automatic scanning microscopes for emulsion data readout and analysis [1,2] made indeed feasible neutrino oscillation experiments based on huge emulsion detectors like CHORUS [3] and OPERA [4].

Nuclear emulsion is a three-dimensional tracking detector made of $\mathrm{AgBr}$ crystals dispersed in a gelatin matrix. The diameter of the $\mathrm{AgBr}$ crystal is $0.2 \mu \mathrm{m}$. A charged particle passing through an emulsion layer ionizes the crystals along its path and produces latent images. After a development procedure, the particle trajectory becomes visible as a line of metallic silver grains. This trajectory is measured with sub-micron position accuracy by using optical microscopes.

\section{Emulsion detector in AEgIS}

The AEgIS [5, 6] experiment at CERN is designed to perform the first precise direct measurement of gravitational interaction between antimatter and matter by detecting the free-fall of a horizontally accelerated cold antihydrogen beam in the Earth's gravitational field. The AEgIS apparatus is installed in one of the extraction points of the Antiproton Decelerator (AD).

The initial goal of the experiment is a $1 \%$ precision measurement for $\Delta \mathrm{g} / \mathrm{g}$. The spatial resolution of the position sensitive detector is a key issue for the success of the experiment. For this reason the employment of emulsion film detectors was considered. Monte Carlo simulations demonstrate indeed that the amount of detected antihydrogen interactions needed to achieve the $1 \%$ precision in the case of $\sim 1 \mu \mathrm{m}$ accuracy for the reconstruction vertex provided by the emulsion is less than 1000, thus obtaning more than one order of magnitude reduction for the required statistics with respect to the employment of silicon strip detector assumed in the original design. The dependency of the precision for the gravity measurement as a function of the number of detected annihilations for various spatial resolutions is shown in Fig. 1.

An intense R\&D was then conducted to define the use of the emulsion technology in the AEgIS apparatus. In particular, suitable working conditions for the employment of emulsion detectors in high vacuum and low temperature were successfully defined [7, 8].

\section{Test beam with antiprotons}

In December 2012 several tests were conducted in the AEgIS beam line at CERN, exposing emulsion detectors to a $\sim 100 \mathrm{keV}$ antiproton beam. The emulsions were installed in a chamber mounted at the position of the future deflectometer for the $\mathrm{g}$ measurement. The main goal was to determine the resolution in reconstructing the $\bar{p}$ annihilation vertex.

After the exposures, the emulsion foils were developed in a dark room at CERN, and then analyzed with the automatic microscope facility of the University of Bern. Thousands of antiproton annihilations were successfully reconstructed. In Fig. 2 (left) the microscope view of an interaction is displayed. The impact parameter distribution for $\bar{p}$ annihilations on bare emulsions and on emulsion covered by a $20 \mu \mathrm{m}$ thick stainless steel foil (simulating the 


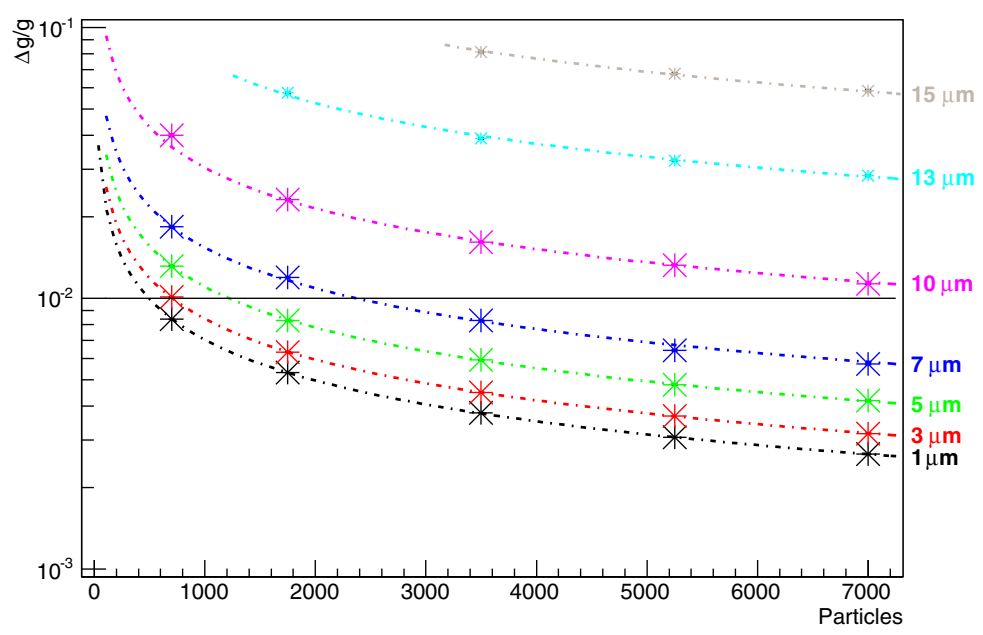

Fig. 1 Simulated precision for the measurement of $g$ as a function of the number of detected annihilation for various vertex resolution of the position sensitive detector. The solid line indicates the goal of the experiment: $1 \%$ for $\Delta \mathrm{g} / \mathrm{g}$. Picture from [8]
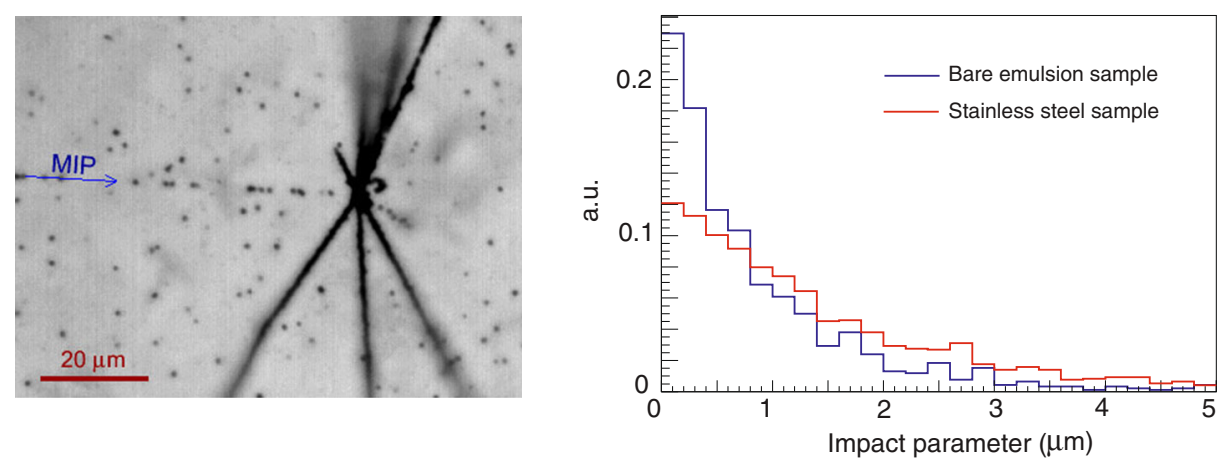

Fig. 2 Microscope view of an antiproton annihilation (left) and impact parameter distribution of interaction products. Pictures from [8]

window needed to separate the deflectometer region from the emulsion detector region in the final experiment) is also shown (right). A resolution of $\sim 1 \mu \mathrm{m}$ was achieved, confirming the feasibilty and the advantages of the employment of emulsion detectors in AEgIS.

The multiplicity distributions of minimum and heavily ionizing particles in antiproton annihilations were also studied. Preliminary results are already providing interesting indications for the discrimination among nuclear models. A publication on this topic is foreseen in a near future.

\section{Proof of principle for the gravity measurement}

The gravity measurement in AEgIS will be performed combining a moiré deflectometer [9] with an emulsion based position sensitive detector. The working principle of such a device 

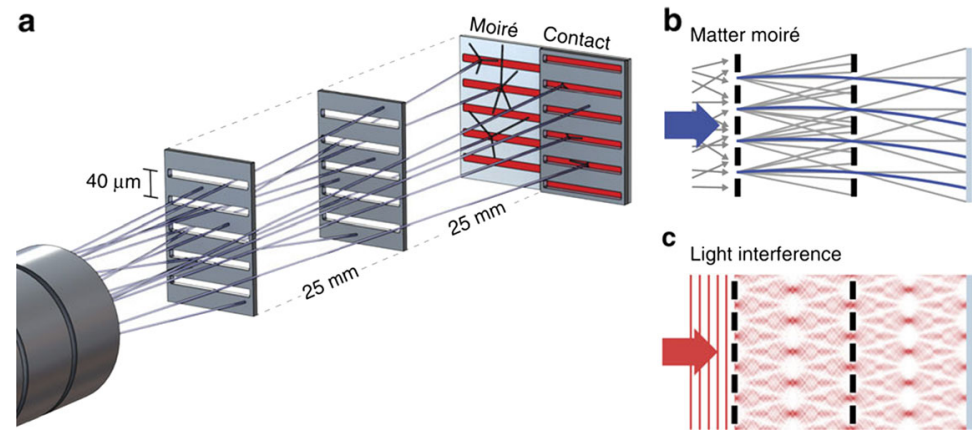

Fig. 3 The moiré deflectometer design (a) and working principle (b) and (c). Picture from [10]
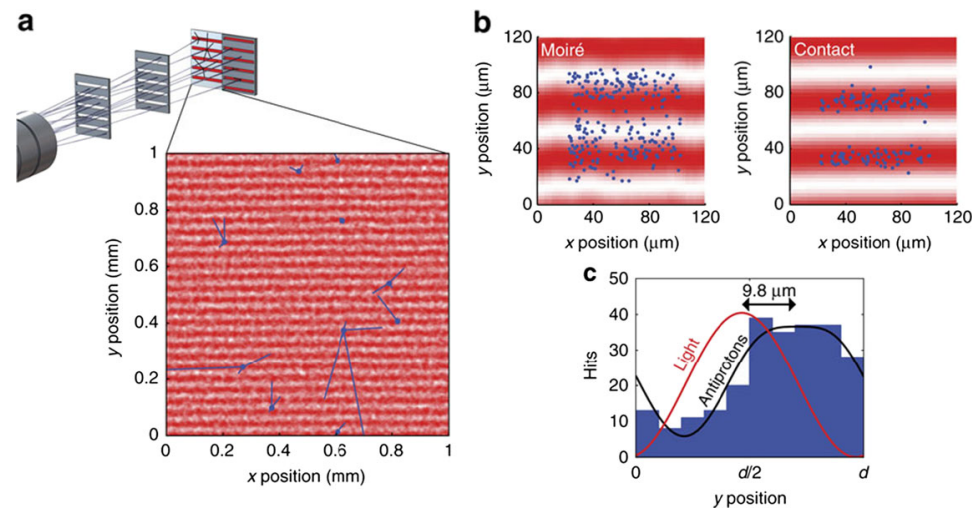

Fig. 4 Shift observed between light and antiprotons patterns with a moiré deflectometer prototipe. Picture from [10]

is shown in Fig. 3. A divergent antihydrogen beam impinges on two subsequent gratings that restrict the transmitted particles to well-defined trajectories. If the distance between gratings and the distance between the second grating and the position sensitive detector are the same, this leads to a shadow fringe pattern which is shifted in the presence of a force. The final position of antihydrogen atoms is determined by reconstructing the trajectories of their annihilation products propagating into the emulsion detector.

A low scale prototipe of such a device was successfully tested with antiprotons during the 2012 test beam campaign [10]. The annihilation vertices of antiprotons were reconstructed and their shifted position was compared with the expected pattern without forces. The comparison was achieved by employing Talbot-Lau interferometry with light. The observed pattern for antiprotons, together with the Talbot-Lau pattern are shown in Fig. 4. By fitting with a proper function, a relative vertical shift between the light and antiproton patterns of $9.8 \pm 0.9 \mu \mathrm{m} \pm 6.4 \mu \mathrm{m}$ was reported. It corresponds to the effect of a force of $F=530 \pm 50 a N \pm 350 a N$ acting on antiprotons during their flight through the deflectometer, compatible with the $\sim 10 \mathrm{G}$ magnetic field measured at that position. 


\section{Conclusions}

Emulsion detectors can measure the position of annihilation vertices of antihydrogen atoms in AEgIS with micrometric precision. Monte Carlo simulations show that the employment of emulsions leads to a one order of magnitude reduction of the data taking time originally foreseen to reach the goal of $1 \%$ uncertainty in the gravity measurement. Furthermore a proof of principle test proved the validity of the AEgIS idea and the reliability of the new design for the downstream region of the apparatus. The AEgIS collaboration looks forward to starting the antihydrogen data collection and analysis.

\section{References}

1. Aoki, S. et al.: Fully automated emulsion analysis system. Nucl. Instr. Meth. B51, 466 (1990)

2. Armenise, N. et al.: High-speed particle tracking in nuclear emulsion by last-generation automatic microscopes. Nucl. Instr. Meth. A551, 261 (2005)

3. Eskut, E. et al.: The CHORUS experiment to search $v_{\mu} \rightarrow v_{\tau}$ oscillation. Nucl. Instr. Meth. A401, 7 (1997)

4. Ereditato, A. et al.: OPERA: an emulsion detector for a long baseline $v_{\mu} \rightarrow v_{\tau}$ oscillation experiment. Nucl. Phys. B66, 1 (1998)

5. Drobychev, G. et al.: http://doc.cern.ch/archive/electronic/cern/preprints/spsc/public/spsc-2007-017.pdf

6. Doser, M. et al.: Exploring the WEP with a pulsed cold beam of antihydrogen. Class. Quantum Grav. 29, 184009 (2012)

7. Amsler, C. et al.: A new application of emulsions to measure the gravitational force on antihydrogen. JINST 8, P02015 (2013)

8. Aghion, S. et al.: Prospects for measuring the gravitational free-fall of antihydrogen with emulsion detectors. JINST 8, P08013 (2013)

9. Oberthaler, M.K. et al.: Inertial sensiting with classical atomic beams. Phys. Rev. A54, 3165 (1996)

10. Aghion, S. et al.: A moiré deflectometer for antimatter. Nat. Comm. 5, 4538 (2014) 